\title{
Foraminoplastic transfacet epidural endoscopic approach for removal of intraforaminal disc herniation at the L5-S1 level
}

\author{
Łukasz Kubaszewski ${ }^{1}$, Jacek Kaczmarczyk ${ }^{1}$, Andrzej Nowakowski ${ }^{2}$, Adam Sulewski ${ }^{2}$ \\ ${ }^{1}$ Orthopedic and Traumatology Department, Poznan University of Medical Science, Poznan, Poland \\ ${ }^{2}$ Spine Surgery, Oncology Orthopedics and Traumatology Department, Poznan University of Medical Science, Poznan, Poland
}

Videosurgery Miniinv (e-pub, ahead of print) DOI: $10.5114 /$ wiitm.2014.40186

\begin{abstract}
Transforaminal endoscopic disc removal in the L5-S1 motion segment of the lumbar spine creates a technical challenge due to anatomical reasons and individual variability. The majority of surgeons prefer a posterior classical or minimally invasive approach. There is only one foraminoplastic modification of the technique in the literature so far. In this paper we present a new technique with a foraminoplastic transfacet approach that may be suitable in older patients with advanced degenerative disease of the spine.
\end{abstract}

Key words: lumbar, foraminal, transforaminal, endoscopic surgery, L5/S1, discectomy.

\section{Introduction}

Percutaneous endoscopic lumbar discectomy (PELD) has become a common procedure since posterolateral percutaneous lumbar disc decompression was presented in the early 1980s [1]. It is also known as transforaminal endoscopic surgery performed in cases of symptomatic lumbar disc herniations as an option for open discectomy.

The advantage of endoscopic procedures is less injury to the paraspinal muscle with a chance for more rapid recovery compared to the classical procedure.

The PELD technique has been developed for upper motion segments of the lumbar spine (L1L5). From the beginning From the beginning problems were bound with approach to the L5/S1 disc, such as high iliac crest or narrow intervertebral foramen [2, 3]. In such cases the PELD procedure is limited to the narrow group of patient without the above-mentioned anatomical obstacles. In the literature we have encountered only one technique modification that deals with the problem, presented by Lee et al., named the foraminoplastic ventral approach [4, 5]. Other techniques for L5/S1 level use modification of the classical approach without foraminoplasty [6]. The majority of surgeons in such cases choose open discectomy, microdiscectomy or endoscopic discectomy through the interlaminar approach [6-8].

The paper presents the technique of the foraminoplastic transfacet epidural approach at the L5-S1 level as a new option to overcome technical problems at this specific motion segment for transforaminal resection of $L 5 / S 1$ disc extrusion with PELD.

\section{Case reports}

Three consecutive cases are reported in which the above-mentioned technique was used.

A retrospective review was performed of 3 consecutive female patients, aged 62-72 years (mean 67.1 years) with extruded disk herniation into the in- 
tervertebral canal unilaterally at the L5-S1 level who underwent decompression by the foraminoplastic transfacet approach between 9.2011 and 10.2012.

Age and comorbidities were the main reason to perform percutaneous decompression. The patients presented with chronic pneumonia $(n=1)$, chronic obstructive pulmonary disease $(n=2)$, diabetes $(n=3)$, or hypertension $(n=2)$.

The mean hospital stay was $48 \mathrm{~h}$ (range: 36-72 h). The inclusion criteria were as follows: extruded disk herniation producing radiating pain, iliac crest with high projection on X-ray examination or narrow foramen hindering approach to the L5-S1 level, no spinal stenosis in computed tomography study, and no response to conservative treatment or to local injections of steroids and lidocaine.

The clinical outcomes were assessed using the visual analog scale (VAS) for leg pain and Oswestry Disability Index (ODI). The mean follow-up period was 12 months (range: 5-18 months).

A preoperative dose of prophylactic antibiotic was administered. The patient was placed prone on a radiolucent operating table under general anesthesia.

The approach trajectory was drawn to enter the center of the L5/S1 intervertebral disc on both anteroposterior (AP) and lateral plane. According to this guideline, the skin entry point was approximately $9-10 \mathrm{~cm}$ aside from the midline as close as possible to the iliac crest under AP view, just to slide over its rim. Under fluoroscopic guidance, a long $2 \mathrm{~mm}$ K-wire was inserted, after skin incision. The K-wire was aiming at the lateral aspect of the L5/S1 facet joint on the affected side and projected through the

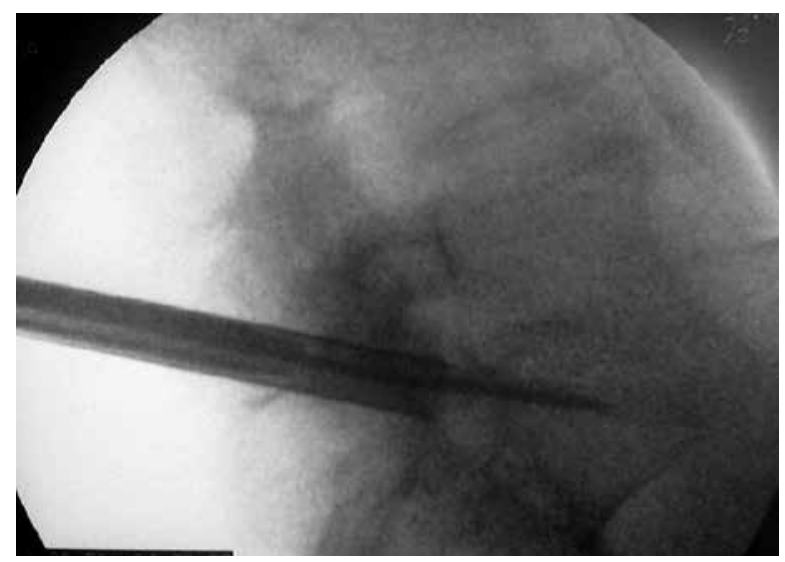

Photo 1. Lateral view of the guide K-wire with obturators centralizing the bone cutter entering the $\mathrm{L} 5 / \mathrm{S} 1$ intervertebral space lower pole of the intervertebral canal. Initially it was guided by hand and after firm fixation against the bony part it was advanced with a power drill. The depth of advancement was confirmed on the lateral view so the tip of the K-wire was in the middle of the intervertebral space in both AP and lateral view. After confirmation of the proper placement a tapered cannula obturator was slid over the wire to the surface of the bone. All the obturators were left over the wire while the bone cutter exceeding the size of working cannula (KARL STORZ GmbH \& Co. KG, Tuttlingen) was slid over it. Obturators allowed centralization of the cutter over the K-wire so the final working channel was centered over initial advancement of the guide wire that was confirmed over its advancement (Photo 1).

The bone cutter was advanced with the help of a hammer due to the strength of the degenerated bone tissue of the facet joint. While advancing it was confirmed on fluoroscopic surveillance to avoid inadvertent K-wire protrusion. The advancement of the cutter was discontinued when the penetration into the intervertebral disc was confirmed on RTG both AP and lateral.

Then the $\mathrm{K}$ wire with cutter and cylindrical section of the facet joint that was stuck between both were removed as a whole (Photo 2).

Then again the K-wire was placed in the elaborated channel with subsequent obturators placement and finally the working side open cannula was introduced into the intervertebral L5/S1 disc space as confirmed with fluoroscopy (Photo 3). The discectomy then was performed in classical manner inside-out with revision of the intervertebral canal where the freed transversing nerve root was visualized (Photo 4).

The mean preoperative VAS score for leg pain was 8.2 (range: 6-10), whereas the mean ODI was $78.5 \%$ (range: 622.2-97.7\%). The average duration of symptoms was 2.3 months (range: 3-12 weeks). At the last follow-up, at least 6 months postoperatively, the mean VAS score for leg pain was 1.8 (range: 0-3.5), whereas the mean ODI was 18.8 (range: 7-34\%). One patient showed excellent and two other good results according to the Macnab outcome classification at the last follow-up examination. All patients were retired and professionally not active. The mean period before return to normal or before disc herniation activity was 2.2 weeks (range: 5 days -4 weeks). No recurrent 


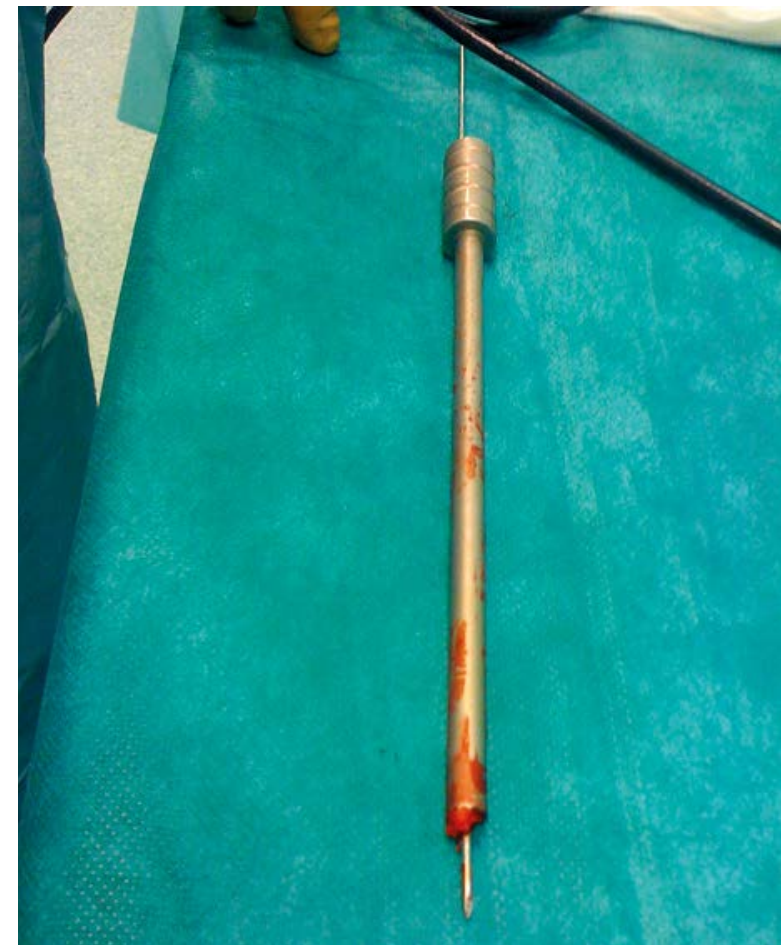

Photo 2. The bone cutter removed with the guiding K-wire and resected part of the facet. Note $\mathrm{K}$-wire is centralized in relation to bone cutter

herniation was observed in the group. We did not observe clinical instability at the operated level. During the study, no patient showed neurological deficit or infection.

\section{Discussion}

Usually in disc pathology at the L5/S1 level interlaminar discectomy or microdiscectomy is performed. Percutaneous endoscopic lumbar discectomy at this segment is generally chosen in younger patients with undisturbed regional anatomy (insignificant collapse of disc space and narrowing of root canal) and proper anatomical conditions (low iliac crest).

Limited possibility to maneuver with the working channel due to the iliac crest makes it extremely difficult to perform the proper direct transforaminal extraction of sequestered disc fragments and their removal from the spinal canal as easily as it is performed at the levels above. In some analyses this level is not even considered for a true lateral approach [9].

In the literature we have found only one modification for PELD surgical technique for L5/S1 level

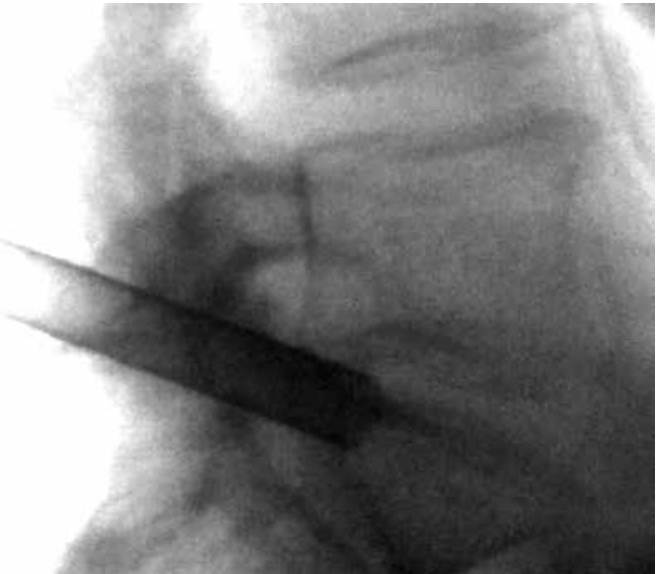

Photo 3. Working cannula placed in the $L 5 / S 1$ intervertebral space

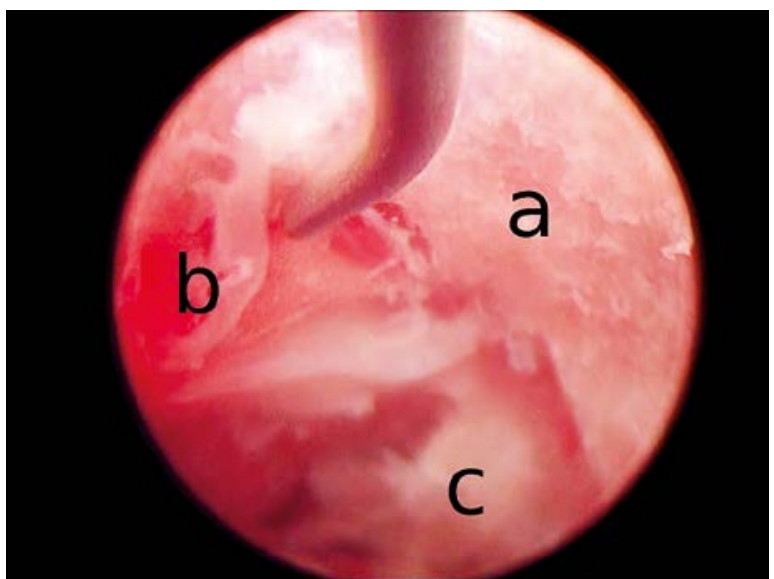

Photo 4. Endoscopic view. The portion of the bone channel performed in the preparation phase (a), decompressed nerve root with perineural fat pointed with the hook (b) with the remainder of the intervertebral disc removed with the endoscope (c)

presented by Lee et al., who proposed a ventral approach. Compared to that technique, the technique presented above has some modifications.

Lee et al. proposed a ventral approach into the intervertebral canal. This approach is suitable for selective resection of the upper facet of S1.

In both techniques the actual cutting is performed with fluoroscopic without visual control. In some surgeons it may evoke anxiety as for risk of neural structure damage during "blind" resection. However, the proper observation of anatomical landmarks makes it possible to perform with minimal risk. 
The main difference is that the Lee et al. technique uses the working cannula as the coordination tool for directing the bone cutter. It is important to note that in this technique neither of the devices, working cannula or bone cutter, is anchored to the bone. Even slight modification of the cutting device position may result in neural insult. Therefore the whole procedure of bone resection should be performed under constant fluoroscopic surveillance. In our technique the initial K-wire is firmly placed through the entire working trajectory - anchored in the bony part of the facet and then introduced to the disc. If it passes through the lower part of the L5/S1 foramen there is no risk of conflict against the nerve root, which is pushed upwards by the protruding disc. Therefore the preparation of the working channel in the facet joint may be performed with appropriate force with help of a hammer, when the bone cutter is centralized with help of the obturators. It guarantees that the K-wire will be the actual center of the working channel. This also makes it possible to shorten the exposure time to irradiation during the procedure.

In our technique all the devices with resected bone have to be removed once the resection is done and then the working cannula has to be put back in the prepared channel. We find it not difficult to follow the working channel as the anatomic landmarks both on the skin and fluoroscopic image help one to easily reestablish the route.

We think that this drawback is comparable or even less problematic for the risk of misplacement of the free-handheld working cannula with bone cutter as in the Lee el al. procedure.

Risk of root injury in the Lee technique is also reduced while the guidewire is placed against the lower part of the intervertebral foramen, then the working cannula is lent against the nerve root causing only its blunt compression.

In our case series the group of patients was older compared to Lee et al. (67.1 and 39.2 respectively). The degenerative process observed in spine results in bone formation over the facet joint as well as vertebral body. In degenerative cases selective ventral resection of the upper S1 facet may be difficult and cause extension of operation time. Furthermore, greater extent of resection is necessary, with the ventral approach, to introduce the working cannula into the disc space with no guarantee that there is enough space to modify the angle of the working cannula if needed.
In the transfacet technique resection is done according to the final trajectory of the working channel. It gives just enough space so the working cannula can be forwarded or retracted with no angular modification. But in the transfacet technique the resection is performed exactly over the spot of the intraforaminally extruded disc, decreasing the risk of "missing" the pathology. Annulotomy and partial resection of the disc is performed also with a bone cutter at the time of working channel preparation. With disc space narrowing and osteophytes at the posterior rim of the vertebral body this seems to be the most rational way to produce the working channel for the L5/S1 intervertebral space in "difficult" anatomy patients. The length of resected bone retrieved from the bone cutter visualizes the extent of bone formation with degenerative changes of the facet joint (range between 3 and $6 \mathrm{~cm}$ in our material). Additionally the trajectory to omit the hypertrophic facet from the lateral side to explore the intervertebral canal would necessitate the trajectory nearly parallel to the frontal plane that is not acceptable at this level.

In the literature the concept of posterolateral endoscopic lumbar nerve decompression is presented as two techniques: indirect central nucleotomy (inside-out, in which fragments are extracted through fenestration of the annulus outside the spinal canal) and direct transforaminal extraction, when sequestered disc fragments are removed from the spinal canal [10]. The transfacet foraminoplastic epidural endoscopic approach is in some sense the combination of both with the additional decompression benefit of foraminoplasty.

As we have presented in this case series, the procedure is quite straightforward with a good clinical outcome and low risk for potential complications.

\section{References}

1. Kambin P, Sampson S. Posterolateral percutaneous suction-excision of herniated lumbar intervertebral discs. Report of interim results. Clin Orthop Relat Res 1986; 207: 37-43.

2. Mirkovic SR, Schwartz DG, Glazier KD. Anatomic considerations in lumbar posterolateral percutaneous procedures. Spine (Phila Pa 1976) 1995; 20: 1965-71.

3. Reulen HJ, Müller A, Ebeling U. Microsurgical anatomy of the lateral approach to extraforaminal lumbar disc herniations. Neurosurgery 1996; 39: 345-50.

4. Lee SH, Kang HS, Choi G, et al. Foraminoplastic ventral epidural approach for removal of extruded herniated fragment at the L5S1 level. Neurol Med Chir 2010; 50: 1074-8. 
5. Lübbers T, Abuamona R, Elsharkawy AE. Percutaneous endoscopic treatment of foraminal and extraforaminal disc herniation at the L5-S1 level. Acta Neurochir (Wien) 2012; 154: 1789-95.

6. Wang B, Lü G, Liu W, et al. Full-endoscopic interlaminar approach for the surgical treatment of lumbar disc herniation: the causes and prophylaxis of conversion to open. Arch Orthop Trauma Surg 2012; 132: 1531-8.

7. Dezawa A, Sairyo K. New minimally invasive discectomy technique through the interlaminar space using a percutaneous endoscope. Asian J Endosc Surg 2011; 4: 94-8.

8. Kim CH, Chung CK. Endoscopic interlaminar lumbar discectomy with splitting of the ligament flavum under visual control. J Spinal Disord Tech 2012; 25: 210-7.

9. Lee DH, Kim NH, Park JB, et al. CT scan assessment of the pathway of the true lateral approach for transforaminal endoscopic lumbar discectomy: is it possible? J Bone Joint Surg Br 2011; 93 1395-9.

10. Nellensteijn J, Ostelo R, Bartels R, et al. Transforaminal endoscopic surgery for symptomatic lumbar disc herniations: a systematic review of the literature. Eur Spine J 2010; 19: 181-204.

Received: 2.04.2013, accepted: 10.08.2013. 